Analysis of a novel stress-sensing technique based on light scattering by an array of birefringent optical waveguides

This content has been downloaded from IOPscience. Please scroll down to see the full text. 2003 J. Opt. A: Pure Appl. Opt. 5 S370

(http://iopscience.iop.org/1464-4258/5/5/394)

View the table of contents for this issue, or go to the journal homepage for more

Download details:

IP Address: 147.96.14.16

This content was downloaded on 30/01/2014 at 17:52

Please note that terms and conditions apply. 


\title{
Analysis of a novel stress-sensing technique based on light scattering by an array of birefringent optical waveguides
}

\author{
J Limeres ${ }^{1}$, M L Calvo ${ }^{1}$, V Lakshminarayanan ${ }^{2}$ and J M Enoch ${ }^{3}$ \\ ${ }^{1}$ Departamento de Óptica, Universidad Complutense de Madrid, 28040-Madrid, Spain \\ ${ }^{2}$ School of Optometry and Department of Physics and Astronomy, University of Missouri, \\ St Louis, MO 63121-4499, USA \\ ${ }^{3}$ School of Optometry, University of California, Berkeley, CA 94720-2020, USA
}

Received 15 November 2002, in final form 5 February 2003

Published 22 August 2003

Online at stacks.iop.org/JOptA/5/S370

\begin{abstract}
We propose a new stress-sensing technique based upon measurement of light scattering produced by an array of birefringent waveguides. When external stress is applied to the array of waveguides, their optical properties are modified via the photoelastic effect. Specifically, the intensity of light scattered by the optical waveguides is significantly affected by stress. In this work we analyse theoretically this stress-induced change. Our numerical simulations show that this effect provides a means to assess the strength and direction of the external force.
\end{abstract}

Keywords: Sensors, fibre optics, fibre waveguides, propagation, scattering and losses

\section{Introduction}

Over the past two decades there have been an increasing number of proposals for producing sensor optical waveguides and to characterize significant parameters such as temperature tolerance and elasticity, and optical properties such as refractive index distribution, for example [1]. With regard to stress sensing, different kinds of optical fibre sensors have been investigated, such as interferometric and grating-based systems. Stress sensors based on modal analysis are very promising [2], but this technique requires characterization of far-field radiation patterns for particular modal orders of the optical fibre. As we shall see, our scattering-based proposal provides a less complicated alternative. With respect to previous work on light scattering applied to stress sensing, it is well known that backward-scattering data provide information on the refractive index distribution and, therefore, changes appearing in this function due to the presence of strains in the structure can be detected by analysing the scattering diagram at $180^{\circ}$ [3]. In this paper we propose a more generalized method based upon the analysis of multiple scattering of light by an array of birefringent optical waveguides.

The basis of our method consists in comparing light scattering before and after an external force is applied. In order to compute multiple scattering by an array of birefringent waveguides, a very general theoretical formulation and the corresponding numerical procedures are required. These powerful computational tools have been developed by the authors in a previous paper [4] and they will be applied here to simulate the stress sensor.

We will consider an external force acting on the array of optical fibres along a given direction. Then we will calculate the new dielectric permittivity tensor via the photoelastic effect and determine the shape of the intensity profile for light scattering. We have observed a variation in this profile compared with multiple scattering in the absence of the action of an external force. Hence the procedure appears to be sensible for determining the direction and magnitude of the external force and the proportional changes in the dielectric permittivity tensor.

The plan of the paper is as follows. In section 2 we provide an overview of the necessary theoretical background. As mentioned above, the details of the model and numerical methods have been presented elsewhere [4]. Section 3 presents the new stress-sensing technique. First, in section 3.1, we introduce the physical principle that allows stress sensing. Then, in section 3.2, we report and discuss the obtained results that support our proposal. Finally, section 4 is devoted to summarizing and drawing the main conclusions of this work. 
Analysis of a novel stress-sensing technique based on light scattering by an array of birefringent optical waveguides

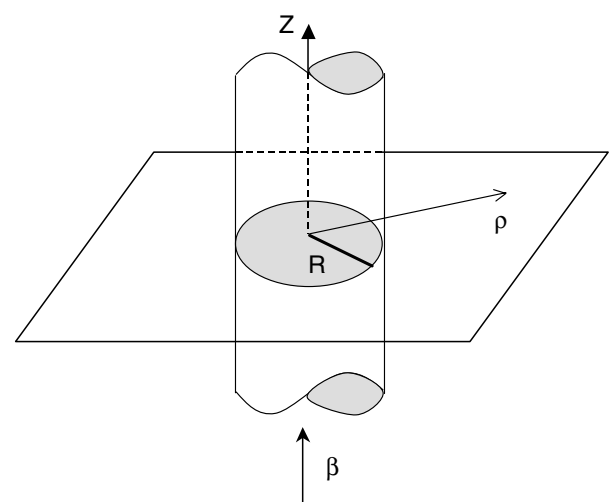

Figure 1. One single-fibre model. $R$ is the radius of each fibre. The wavevector of the incoming radiation, $\beta$, is defined at the $Z$-direction (parallel to the optic axis of the waveguide). The magnetic field is plane polarized at the $X Y$-plane.

\section{Theoretical background}

\subsection{Light scattering by a cylindrical birefringent waveguide}

Let us consider a cylindrical optical waveguide (optical fibre) of radius $R$ placed within an infinite, isotropic, homogeneous medium. Let the fibre axis be the $Z$-axis of our coordinate system as shown in figure 1 . The symbol $\rho$ will denote the position of points belonging to the $X Y$-plane. In this work we have considered homogeneous birefringent waveguides with uniaxial symmetry, their optical axis being parallel to the $Z$ direction. Consequently, the dielectric permittivity tensor $\varepsilon$ inside the fibre is diagonal and has only two independent components: $\varepsilon_{33}$, associated with propagation along the $Z$ axis, and $\varepsilon_{11}$, corresponding to the $X Y$-plane, since $\varepsilon_{11}=\varepsilon_{22}$.

We have investigated the scattering of a monochromatic linearly polarized light wave propagating along the $Z$-direction. Let the incident magnetic field of light, $h^{(0)}$, lie on the $X Y$-plane. In our calculations we have followed the eikonal formulation of light scattering and we have kept the first-order approximation, acceptable for weak scattering processes [5]. Such an approach leads to the following integral equation (see [6] for further details):

$h(\rho)=h^{(0)}+\int_{\Omega} \mathrm{d}^{2} \rho^{\prime}\left[\frac{1}{4 \mathrm{i}} H_{0}^{(1)}\left(\rho, \rho^{\prime}\right) \hat{P}_{1}\left(\rho^{\prime}\right)+\hat{P}_{2}\left(\rho, \rho^{\prime}\right)\right] h^{(0)}$,

where $H_{0}^{(1)}$ is a Hankel function of the first kind and zero-order and $\hat{P}_{1}$ and $\hat{P}_{2}$ are matrix functions depending on the dielectric permittivity tensor and the second derivatives of $H_{0}^{(1)}$ (see [6]). The first term in equation (1) is simply the incident wave amplitude $h^{(0)}$. The second contribution is the scattered field amplitude, a complex quantity that depends on the properties of the light and the scatterer.

\subsection{Mechanical-optical effects}

When an external force is applied to an optical waveguide, an elastic deformation occurs. Then, its birefringence is modified through the so-called photoelastic effect [7]. The mathematical formulation of this phenomenon can be accomplished by introducing a modified dielectric permittivity tensor $\tilde{\varepsilon}$ that

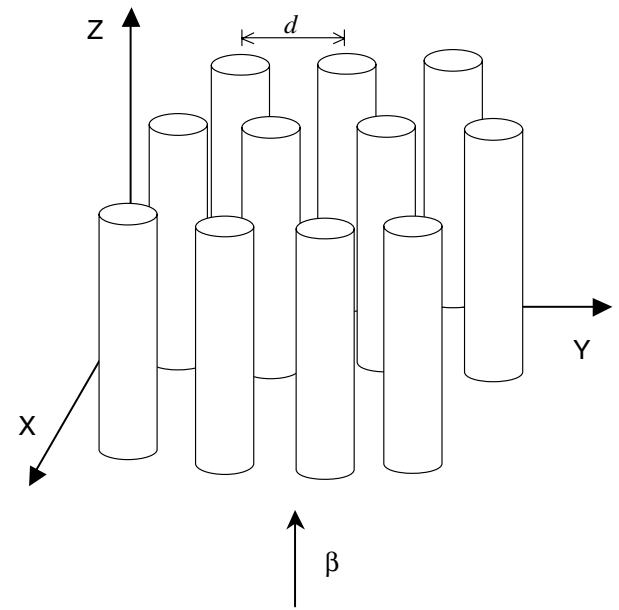

Figure 2. $N$ coupled waveguides. $d$ is the average distance between two adjacent (parallel) fibres. The impact plane $X Y$ defines the plane where the amplitude of the light scattered by the fibre array is determined (see text for details).

takes into account the photoelastic effect through some additional terms in the following way [8]:

$$
\tilde{\varepsilon}_{i k}=\varepsilon_{e} \delta_{i k}+a_{1} u_{i k}+a_{2} u_{l l} \delta_{i k}
$$

where $\delta_{i k}$ is the Kronecker delta, $a_{1}$ and $a_{2}$ are the elasticoptical constants of the medium and $u_{i j}$ are the components of the stress tensor, which are proportional to the transverse force per unit length applied to each waveguide.

\subsection{Generalization to an array of birefringent waveguides}

Let us now consider an array of parallel cylindrical waveguides (see figure 2). We will apply the sampling theorem [9] to generalize the above-obtained single-fibre results to a bundle of $N$ fibres. Notice that the scattered field amplitude produced by a single cylindrical waveguide shows cylindrical symmetry. Hence, the total field amplitude, $h_{T O T}$, scattered by an array of fibres of radius $R$ can be written as [10]:

$$
\begin{aligned}
h_{\text {TOT }} & =\frac{\pi}{4} \sum_{m} \sum_{n} h_{i}(n d / 2, m d / 2) \\
& \times \frac{J_{1}\left(2 \pi / d \sqrt{(x-n d / 2)^{2}+(y-m d / 2)^{2}}\right)}{2 \pi / d \sqrt{(x-n d / 2)^{2}+(y-m d / 2)^{2}}},
\end{aligned}
$$

where $h_{i}$ is the scattered field amplitude corresponding to each single fibre and $J_{1}$ is a first-order Bessel function. The summation indices $n$ and $m$ label the sampling spatial points, $x_{n}=n d / 2, y_{m}=m d / 2, d$ being the distance between the centres of two adjacent fibres. Depending on the geometrical arrangement of the array, different sampling lattices may be used.

\section{Results and discussion}

\subsection{Physical basis of the stress-sensing technique}

In this work we are dealing with a uniaxial medium, meaning that it presents two refractive indices: extraordinary, for light polarized along the optical axis (the $Z$-axis), and ordinary, for $X Y$-plane polarization. That is, directions $X$ and $Y$ are 


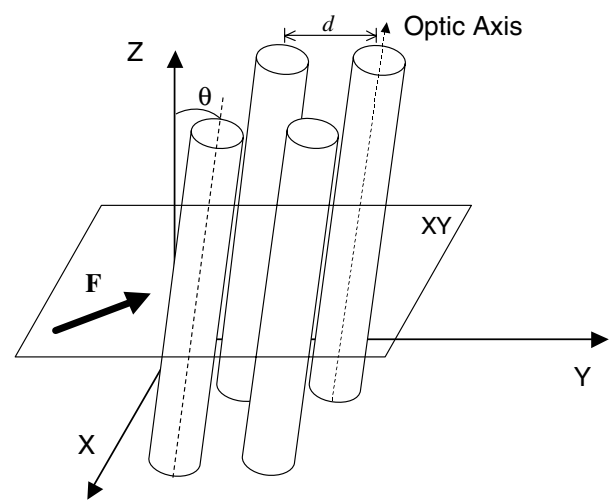

Figure 3. A model for the mechanical-optical effect: an external force $F$ is applied and defined in some arbitrary $X Y$-plane. The conditions of incidence of light are similar to those in figures 1 and 2 . Notice that in this work we are considering arrays for which the angle $\theta$ is small.

optically indistinguishable. Hence, components $X$ and $Y$ of our incident light beam will undergo exactly the same scattering process and no difference between either component will be detectable.

When the array of fibres is subjected to stress under an external force or pressure (see figure 3), its index tensor is modified through the photoelastic effect, as explained above Consequently, the symmetry of the $X Y$ plane is broken because now $\varepsilon_{11} \neq \varepsilon_{22}$, i.e. both directions $X$ and $Y$ are no longer optically indistinguishable. With regard to light scattering, this results in a change in the relative behaviour of both components of the scattered light field. Given the relative variation in the scattered field amplitude, the induced birefringence $\varepsilon_{11}-\varepsilon_{22}$ can be directly evaluated, which allows us to calculate the direction and strength of the applied external force through formula (2). This is the basis of our proposal for a novel stress sensor.

\subsection{Results and discussion}

In order to assess the effect induced on the waveguide by an external force, we have proceeded as follows. First, we have integrated equation (1) by Gaussian quadratures [11] to obtain the light field scattered by a single birefringent waveguide. Then, we have used equation (3) to compute the total scattering field amplitude corresponding to an array of cylindrical waveguides in the absence of stress. After the application of an external force, the components of the dielectric permittivity tensor have been recalculated through equation (2). Finally, we have used this modified tensor $\varepsilon^{\prime}$ to obtain the new shape of the light scattering intensity profile.

In our calculations we have considered an array of seven fibres of radius $R=0.75 \mu \mathrm{m}$, separated by a distance $d=2 \mu \mathrm{m}$. The small size of the optical fibres has been chosen in order to enhance the sensitivity of the stress sensor. Notice that the photoelastic effect is proportional to the transverse force (see equation (2) and explanation below), which in turn is inversely proportional to the transverse area of the fibre. The birefringent waveguiding medium exhibits an ordinary refractive index $n_{o}=1.34$ and an extraordinary refractive index $n_{e}=1.35$. These values correspond to refractive

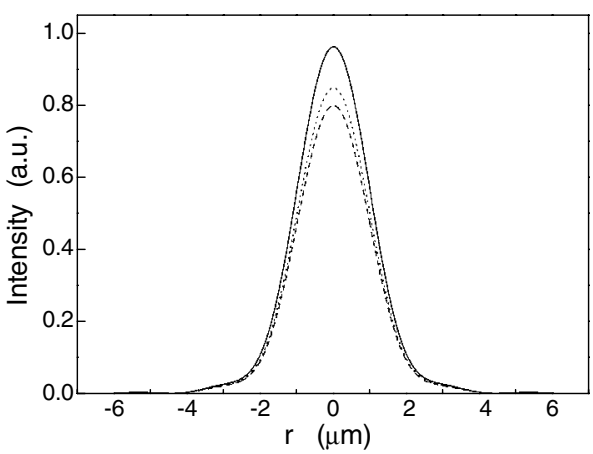

Figure 4. Spatial dependence of the forward-scattering light intensity. Dotted-curve: zero applied force, $F_{y}=0$. Dashed curve: $F_{y}=0.01 \mathrm{~N}, X$-polarized light. Solid curve: $F_{y}=0.01 \mathrm{~N}$, $Y$-polarized light.

indices of biological material, in order to analyse the stress effect on biological waveguides. Notice, however, that our model is not restricted to these particular waveguides but can be applied to various types. Photoelastic constants have been taken from available data from related experiments and are a good approach for the purposes of our analysis. Thus, for the photoelastic constants $a_{1}$ and $a_{2}$, we have considered typical values of silica fibres $a_{1}=0.38 \times 10^{-12} \mathrm{~m}^{2} \mathrm{~N}^{-1}$ and $a_{2}=2.68 \times 10^{-12} \mathrm{~m}^{2} \mathrm{~N}^{-1}$.

Without loss of generality, we have chosen an external force applied along the $Y$-axis. First, we have compared the results obtained under zero external force $\left(F_{y}=0\right)$ with the results after application of a very weak force $\left(F_{y}=0.01 \mathrm{~N}\right)$. For zero external force, scattering profiles for $X$-polarized and $Y$-polarized light are identical because of the axial symmetry of the physical system. However, this symmetry is broken after application of an external force, as stated in section 3.1. After stress is induced, the intensity of $X$-polarized light decreases slightly (see figure 4, dashed curve) while the $Y$-polarized light is enhanced (see figure 4, solid curve). Notice that the latter coincides with the direction of application of the external force. This loss of symmetry could be detected by monitoring the light field amplitude after scattering. Therefore, analysis of the scattering profile will allow us to determine the direction of application of the force (i.e. stress). It should be noted that even for a very weak force $(F=0.01 \mathrm{~N})$ the difference in intensity between both polarizations is $\sim 20 \%$.

From figure 4 we can also state that the major impact of stress occurs at the central peak of the scattered light profile, which corresponds to forward scattering. This is very convenient for practical purposes since forward-scattering observation could be easily performed. We have calculated the dependence of forward scattering on the strength of the applied force. Figure 5 shows the forward-scattering intensity for $X$ - (dashed curve) and $Y$ - (solid curve) polarized light as a function of the strength of the force, $F_{y}$, in the range $0<F_{y}$ $<0.1 \mathrm{~N}$. Notice that the difference between both components is enhanced as $F$ becomes stronger. For the highest values of $F_{y}$, the intensity of $X$-polarized light is strongly suppressed. Even for moderate values of force, such as $F_{y}=0.05 \mathrm{~N}$, a difference around $60 \%$ between both polarizations is observed. Since the amount of change for both light polarizations is directly related to the strength of the applied force, figure 5 
Analysis of a novel stress-sensing technique based on light scattering by an array of birefringent optical waveguides

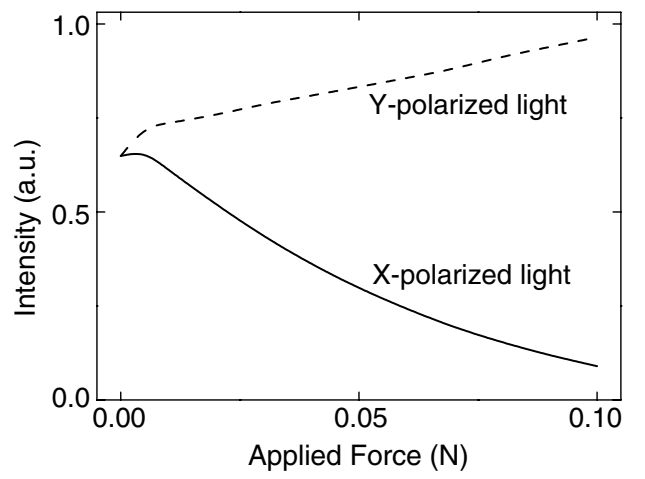

Figure 5. Forward-scattering intensity against the strength $F_{y}$ of the applied force. The solid curve corresponds to $X$-polarized light and the dotted curve, to $Y$-polarized light.

provides a calibration curve for our stress-sensing system. We note here that for different values of the refractive index, for instance those corresponding to silica fibres $(n \sim 1.52)$, we obtain a behaviour (not displayed here for brevity) similar to the one shown in this figure 5, although the stress-induced effect is less enhanced.

\section{Conclusions}

In this paper we have evaluated the possibility of implementing a stress sensor based on light scattering by an array of birefringent waveguides. To this end we have calculated the impact of stress on the intensity profile of scattered light.

Our results show a significant change in the profile of the field. The change especially affects the central peak of the intensity profile which corresponds to forward scattering. This is very profitable for applications since forward-scattering measurements are more easily accomplished.

One main consequence of stress is the loss of axial symmetry due to the appearance of two privileged directions: the axis parallel to the force ( $Y$-axis) and the perpendicular axis $(X$-axis). The asymmetry induced by stress between $X$ - and $Y$-polarized light provides a method to assess the direction of application of the external force.

We have also found that the stress-induced difference between $X$ - and $Y$-polarized light is strongly dependent on the strength of the applied force. We have plotted this dependence for both polarizations, thus obtaining a 'calibration curve' for our stress-sensing system.
Finally, the technique offers good sensitivity, since even for very weak forces $(F=0.01 \mathrm{~N})$ a significant difference of $\sim 20 \%$ between $X$ - and $Y$-polarized light can be observed

We conclude that the effect of stress on scattering by an array of birefringent waveguides could be the basis of an optical stress sensor.

\section{Acknowledgments}

The authors gratefully acknowledge financial support from the 'New Del Amo foundation' under the New Del Amo Joint Academic Project (University of California-Universidad de Madrid) and from the Universidad Complutense de Madrid under Multidisciplinary Project PR486/97-7477/97.

Partial results were presented at the ICO-19th Conference on 'Optics for the Quality of Life' (Florence, Italy, 25-31 August 2002)

\section{References}

[1] Grattan K T V and Sun T 2000 Fiber optic sensor technology: an overview Sensors Actuators 82 40-61

[2] Wolinski T R 2000 Polarimetric optical fibres and sensors Progress in Optics vol 40, ed E Wolf (Amsterdam: Elsevier) ch I

[3] Cordero Ianarella R F 1980 Transversely incident beam wave scattering from inhomogeneous fibres J. Opt. Soc. Am. 70 799-804

[4] Limeres J, Calvo M L, Lakshminarayanan V and Enoch J M 2003 Light scattering by an array of birefringent optical waveguides. Theoretical foundations J. Opt. Soc. Am. B at press

[5] Sharma S K and Somerfeld D J 1999 Scattering of light in the eikonal approximation Progress in Optics ed E Wolf (Amsterdam: Elsevier) ch III

[6] Alvarez-Estrada R F and Calvo M L 1983 Single-mode anisotropic cylindrical dielectric waveguides Opt. Acta $\mathbf{3 0}$ 481-503

[7] Dändliker R 1992 Rotational effects of polarization in optical fibres Anisotropic and Non-Linear Optical Waveguides ed C G Someda and G Stegman (Amsterdam: Elsevier) p 42 section 2.2

[8] Landau L D and Lifshitz E M 1960 Electrodynamics of Continuous Media (Oxford: Pergamon) ch XI section 81

[9] Bakarat R 1964 Application of the sampling theorem to optical diffraction theory J. Opt. Soc. Am. 54 921-30

[10] Goodman J W 1996 Introduction to Fourier Optics 2nd edn (New York: McGraw-Hill) ch 2 section 2.4

[11] Press W H, Flannery B P, Teukolsky S A and Vetterling W T 1988 Numerical Recipes in C: the Art of Scientific Computing (Cambridge: Cambridge University Press) 